\title{
Multiscale Validation of the Applicability of Micromechanical Models for Asphalt Mixture
}

\author{
Jiupeng Zhang, ${ }^{1}$ Zepeng Fan, ${ }^{1}$ Jianzhong Pei, ${ }^{1}$ Rui Li, ${ }^{1}$ and Mingfeng Chang ${ }^{2}$ \\ ${ }^{1}$ School of Highway, Chang'an University, Shaanxi, Xian 710064, China \\ ${ }^{2}$ School of Materials Science and Engineering, Chang'an University, Shaanxi, Xian 710064, China \\ Correspondence should be addressed to Jianzhong Pei; peijianzhong@126.com
}

Received 22 April 2015; Revised 22 July 2015; Accepted 28 July 2015

Academic Editor: Antônio G. B. de Lima

Copyright (c) 2015 Jiupeng Zhang et al. This is an open access article distributed under the Creative Commons Attribution License, which permits unrestricted use, distribution, and reproduction in any medium, provided the original work is properly cited.

\begin{abstract}
Asphalt mixture is more complicated than other composite materials in terms of the higher volume fraction of aggregate particles and the viscoelastic property of asphalt matrix, which obviously affect the applicabilities of the micromechanical models. The applicabilities of five micromechanical models were validated based on the shear modulus of the multiscale asphalt materials in this paper, including the asphalt mastic, mortar, and mixture scales. It is found that all of the five models are applicable for the mastic scale, but the prediction accuracies for mortar and mixture scales are poorer. For the mixture scale, all models tend to overestimate at the intermediate frequencies but show good agreement at low and high frequencies except for the Self-Consistent (SC) model. The Three-Phase Sphere (TPS) model is relatively better than others for the mortar scale. The applicability of all the existing micromechanical models is challenged due to the high particle volume fraction in the multiscale asphalt materials as well as the modulus mismatch between particles and matrix, especially at the lower frequencies (or higher temperatures). The particle interaction contributes more to the stiffening effect within higher fraction than $30 \%$, and the prediction accuracy is then deteriorated. The higher the frequency (or the lower the temperature) is, the better the model applicability will be.
\end{abstract}

\section{Introduction}

Asphalt mixture is a kind of heterogeneous composite material, consisting of asphalt binder and mineral aggregates with different sizes. The traditional researches on the mechanical properties of asphalt mixture and its failure mechanism are mostly based on the continuum mechanics theory and the experimental method. However, the mechanical properties of asphalt mixture are closely related to the complicate internal structure, which is dependent on the various raw materials' properties, shapes, sizes, and proportions. The traditional analyzing methods fail to reveal the microstructure related failure mechanism of asphalt mixture, such as the formation and propagation of microcracks, the micro damage caused due to the heterogeneous material, and the local failures caused due to the stress concentration. Recently, researchers have realized the importance of the internal microstructure to the macromechanics properties, and the micromechanics theory of composites has been introduced to research the macromechanical properties from the meso- and microscale [1-4].

At the microscale, the asphalt mixture could be considered as a kind of heterogeneous multiphase composite which is composed of asphalt binder, aggregates, asphaltaggregate interphase, microcrack, and void. The homogenization approach of the heterogeneous material is a fundamental problem in the area of composite, which mainly involves the prediction of the effective modulus of the composite. Micromechanical modeling techniques have long been successfully used to predict the effective modulus from mechanical properties and volume fractions of individual constituents for composite materials such as metal and polymer matrix composites [5-9]. For asphalt mixture, the homogenization method could be applied to acquire the effective properties, like the effective modulus. However, compared with other composites, asphalt mixture exhibits some special characteristics both due to the complicated microstructure and mechanical properties of raw materials. Firstly, the aggregates 
play the role of reinforcement phase in this composite, and its volume fraction is usually up to $80 \%$, which is far higher than the reinforcement phase in other composite materials. Secondly, the aggregates in common asphalt mixtures are with various sizes from $0.075 \mathrm{~mm}$ up to $37.5 \mathrm{~mm}$ while the reinforcement phase is with a relatively uniform size in other composite materials. Well-graded aggregates obviously exhibit the size effect, which means aggregate with different size plays different role in this composite $[10,11]$. Moreover, asphalt binder is a viscoelastic matrix of this composite material, which also affects the asphalt-aggregate interphase, and causes more difficulties to the applications of these micromechanical models $[12,13]$.

Though considerable achievements have been made by previous researchers, few have involved the applicability of the existing micromechanical models on asphalt mixture. So, in this paper, the micromechanical models for composite materials were firstly introduced and evaluated. Then, these models were applied to predict the mechanical properties of asphalt materials from the multiscales and compared with the experiment results to validate the applicability of micromechanical models. In addition, the effects of particle volume fraction and modulus mismatch between reinforcement phase and matrix phase were also analyzed from the mastic scale.

\section{Literature Review on the Micromechanical Models}

Since Eshelby's pioneering work [14] on elastic solutions for an infinite medium including a single inclusion, a lot of micromechanical models have been developed in the past decades, including the Self-Consistent (SC) model, the MoriTanaka (M-T) model, the Generalized Self-Consistent (GSC) model, the Differential Scheme Effective Medium (DSEM) model, and the Three-Phase Sphere (TPS) model.

In the SC model, any material point on the particles is isolated as an infinitesimal volume element. Then the rest of the material is homogenized as the uniform material, whose mechanical property is identical to the composite itself $[5,6$, 12 ]. The effective bulk modulus $K$ and shear modulus $G$ of the $\mathrm{SC}$ model can be written as

$$
\begin{aligned}
& K=K_{0}+\frac{c\left(K_{1}-K_{0}\right)(3 K+4 G)}{3 K+4 G}, \\
& G=G_{0}+\frac{5 c\left(G_{1}-G_{0}\right) G(3 K+4 G)}{6 G_{1}(K+2 G)+G(9 K+8 G)},
\end{aligned}
$$

where $c$ denotes the volume fraction of particles and $K$, $G ; K_{0}, G_{0}$; and $K_{1}, G_{1}$ represent the bulk modulus and shear modulus of effective medium, matrix, and particle, respectively.

The M-T model involves complicated manipulation of the field variables in Mori and Tanaka [7]. In this method, a particle is embedded into the matrix with a uniform strain same as the matrix's averaged strain, and the particle's averaged strain is derived from the solution for single particle

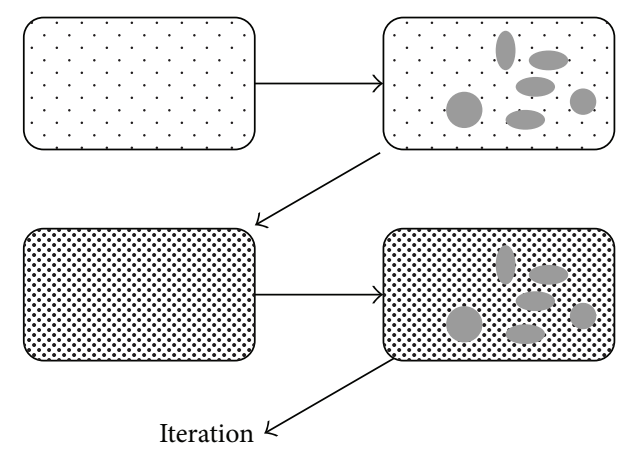

FIGURE 1: Schematic diagram of the differential scheme method.

embedded in the infinite matrix [12]. The effective bulk modulus $K$ and shear modulus $G$ can be expressed as

$$
\begin{aligned}
K & =K_{0}+\frac{c K_{0}}{K_{0} /\left(K_{1}-K_{0}\right)+3(1-c) K_{0} /\left(3 K_{1}+4 G_{0}\right)}, \\
& =G_{0} \\
& +\frac{c G_{0}}{G_{0} /\left(G_{1}-G_{0}\right)+6(1-c)\left(K_{0}+2 G_{0}\right) / 5\left(3 K_{1}+4 G_{0}\right)} .
\end{aligned}
$$

In the GSC model, the spherical particle is embedded in a concentric spherical shell of the matrix material. Shell and particle dimensions are chosen to correspond to the prescribed volume fraction, and the particle-shell assembly in turn is embedded in an infinite medium with unknown effective properties [15]. The solution for effective shear modulus $G$ is the solution of a quadratic equation

$$
A\left(\frac{G}{G_{0}}\right)^{2}+2 B\left(\frac{G}{G_{0}}\right)+C=0 .
$$

The expressions for $A, B$, and $C$ can be found in Christensen and Lo [8]. Shashidhar and Shenoy [16] provided a simplification form of the GSC model that is suitable for mastic. The effective bulk modulus $K$ is the same as the M-T model.

By introducing the Differential Scheme Effective Medium theory into the micromechanics field, Mclaughlin [9] and Norris [17] successfully developed the differential method. In this method, the composite material is viewed as a sequence of dilute composites to which an increment of inclusions is added, as shown in Figure 1. This method shows great potential in predicting the effective properties of composites with high particle volume fraction. Shu and Huang [18] and Kim and Buttlar [19] further derived new models suit for asphalt mixture, respectively, and only the latter is given in this research. The effective bulk modulus $K$ and shear modulus $G$ can be written as

$$
\begin{aligned}
& K=K_{1}-(1-c)\left(K_{1}-K_{0}\right)\left[\frac{3 K+4 G}{3 K_{0}+4 G_{0}}\right], \\
& G=G_{1}-(1-c)\left(G_{1}-G_{0}\right)\left[\frac{G}{G_{0}}\right]^{2 / 5}\left[\frac{3 K+4 G}{3 K_{0}+4 G_{0}}\right]^{1 / 5} .
\end{aligned}
$$

To take the effect of particle size and aggregate gradation on the asphalt mixture modulus into consideration, Li et al. 


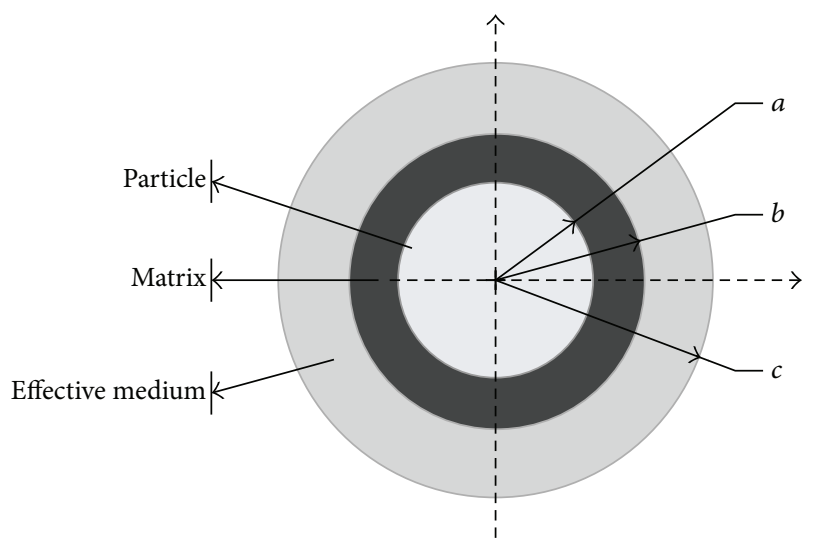

FIgURE 2: Three-Phase Sphere model.

[20] proposed a two-layer built-in micromechanical model and derived an equation predicting the effective modulus of asphalt mixture at 2D situation. Shu and Huang [21, 22] extended this model to the $3 \mathrm{D}$ situation and investigated the dynamic modulus of asphalt mixture. The research here named this series of models the Three-Phase Sphere model and the schematic diagram is shown in Figure 2. The estimate formula of the effective elastic modulus can be written as

$$
\begin{aligned}
& E(a) \\
& =\frac{E_{0}(1-n)(1-2 \nu)}{x_{1}-9 E_{1} n\left(1-v_{0}\right)^{2} /\left(4 E_{0}(1-n)\left(1-2 v_{1}\right)+4 E_{1} x_{2}\right)},
\end{aligned}
$$

where $n=a^{3} / b^{3}, x_{1}=1 / 2 n\left(1+v_{0}\right)+\left(1-2 v_{0}\right), x_{2}=$ $1 / 2\left(1+v_{0}\right)+n\left(1-2 v_{0}\right), a=$ radius of the particle, $b-a=$ thickness of the matrix, $c-b=$ thickness of the surrounding equivalent medium, $c$ = radius of equivalent medium, and $\nu, v_{0}$, and $\nu_{1}$ represent Poisson's ratio of effective medium, matrix, and particle, respectively; more details can be seen in Shu and Huang $[21,22]$.

Those models were derived from elastic theory, but not limited to elastic materials, which could be extended to viscoelastic solutions based on the elastic-viscoelastic correspondence principle [23]. According to the correspondence principle, the effective complex modulus of the composite can be obtained by replacing the elastic modulus with its Carson transformation in the Laplace domain [23, 24]. For asphalt mixtures, the particle reinforcement phase (aggregate and void) can be considered elastic while the matrix phase (binder, mastic, mortar, and mixture) can be considered viscoelastic. By replacing the elastic, bulk, and shear modulus with their corresponding transformed form in (1) through (6), the predictive equations were finally established.

\section{Multiscale Validations of the Micromechanical Models}

3.1. Experiments of the Multiscale Asphalt Materials. According to the research reported by Underwood and Kim [25], the asphalt mixture is a multiscale composite material, and it
TABLE 1: The aggregate gradations for asphalt mastic, mortar, and

\begin{tabular}{|c|c|c|c|}
\hline \multirow{2}{*}{ Sieve size $(\mathrm{mm})$} & \multicolumn{3}{|c|}{$\%$ passing } \\
\hline & Mixture & Mortar & Mastic \\
\hline 25.4 & 100 & & \\
\hline 19 & 100 & & \\
\hline 12.5 & 100 & & \\
\hline 9.5 & 96 & & \\
\hline 4.75 & 66 & & \\
\hline 2.36 & 48 & 100 & \\
\hline 1.18 & 37 & 77 & \\
\hline 0.6 & 30 & 63 & \\
\hline 0.3 & 21 & 44 & \\
\hline 0.15 & 11 & 23 & \\
\hline 0.075 & 5.8 & 12.1 & 100 \\
\hline 0.030 & 3.3 & 6.9 & 57 \\
\hline 0.023 & 2.9 & 6.0 & 50 \\
\hline 0.017 & 2.1 & 4.3 & 36 \\
\hline 0.011 & 1.5 & 3.2 & 27 \\
\hline 0.0077 & 1.2 & 2.5 & 21 \\
\hline 0.0038 & 0.9 & 1.8 & 15 \\
\hline 0.0036 & 0.7 & 1.5 & 12 \\
\hline 0.0029 & 0.6 & 1.2 & 10 \\
\hline 0.0025 & 0.6 & 1.2 & 10 \\
\hline 0.0023 & 0.6 & 1.2 & 10 \\
\hline 0.0013 & 0.4 & 0.8 & 7 \\
\hline
\end{tabular}
mixture.

\begin{tabular}{|c|c|c|c|c|}
\hline $\begin{array}{l}\text { Multiscale } \\
\text { materials }\end{array}$ & Matrix & Particles & Volume fraction & $\begin{array}{c}\text { Poisson } \\
\text { ratio }\end{array}$ \\
\hline $\begin{array}{l}\text { Asphalt } \\
\text { binder }\end{array}$ & - & - & - & 0.495 \\
\hline $\begin{array}{l}\text { Asphalt } \\
\text { mastic }\end{array}$ & Binder & Fillers & 0.26 & 0.495 \\
\hline $\begin{array}{l}\text { Asphalt } \\
\text { mortar }\end{array}$ & Mastic & $\begin{array}{c}\text { Fine } \\
\text { aggregates } \\
\text { and voids }\end{array}$ & 0.65 and 0.065 & 0.35 \\
\hline $\begin{array}{l}\text { Asphalt } \\
\text { mixture }\end{array}$ & Mortar & $\begin{array}{c}\text { Coarse } \\
\text { aggregates }\end{array}$ & 0.43 & 0.35 \\
\hline
\end{tabular}

TABLE 2: Multiscale materials properties and proportions.

could be divided into four scales, which are binder scale, mastic scale, mortar scale, and mixture scale. The asphalt mastic is the composite materials consisting of asphalt binder and fillers (aggregates smaller than $0.075 \mathrm{~mm}$ ), asphalt mortar is the composite materials consisting of asphalt mastic, fine aggregates (aggregates larger than $0.075 \mathrm{~mm}$ but smaller than $2.36 \mathrm{~mm}$ ), and voids, and asphalt mixture is the composite materials consisting of asphalt mortar and coarse aggregates (aggregates larger than $2.36 \mathrm{~mm}$ ). The aggregate gradations for asphalt mastic, mortar, and mixture are shown in Table 1. The aggregates are elastic materials, and their shear modulus and Poisson's ratio could be assumed as 19,000 MPa and 0.25, respectively $[26,27]$. The multiscale compositions and the material properties are shown in Table 2. 
TABLE 3: Test methods of the multiscale asphalt materials.

\begin{tabular}{lccc}
\hline Material scale & Temperatures $\left({ }^{\circ} \mathrm{C}\right)$ & Frequencies $(\mathrm{Hz})$ & Mode of loading \\
\hline Binder and mastic & $10,16,19,22,25,30,40,54$ & $14,6.5,3.0,1.4,0.65,0.30,0.14,0.10$ & $\begin{array}{c}\text { Fully reversed oscillatory shear test } \\
\text { (parallel plate) }\end{array}$ \\
Mortar & $14,23,38,58$ & $14,6.5,3.0,1.4,0.65,0.30,0.14,0.10$ & $\begin{array}{c}\text { Fully reversed oscillatory shear test } \\
\text { (torsional cylinder) } \\
\text { Mixture }\end{array}$ \\
\hline
\end{tabular}

TABLE 4: Fitting parameters of the master curves.

\begin{tabular}{lccccc}
\hline $\begin{array}{l}\text { Multiscale } \\
\text { materials }\end{array}$ & $\alpha$ & $\beta$ & $f_{0}$ & $p$ & $R^{2}$ \\
\hline $\begin{array}{l}\text { Asphalt } \\
\text { binder }\end{array}$ & -1.57 & 9.14 & -3.17 & 0.143 & 0.99 \\
$\begin{array}{l}\text { Asphalt } \\
\text { mastic }\end{array}$ & -0.68 & 9.29 & -3.38 & 0.151 & 0.99 \\
$\begin{array}{l}\text { Asphalt } \\
\text { mortar }\end{array}$ & 6.35 & 9.85 & -2.33 & 0.230 & 0.99 \\
$\begin{array}{l}\text { Asphalt } \\
\text { mixture }\end{array}$ & 6.76 & 10.08 & -2.12 & 0.187 & 0.99 \\
\hline
\end{tabular}

The asphalt binder is a viscoelastic material, which makes the asphalt mastic, asphalt mortar, and asphalt mixture be the viscoelastic materials. So, to represent the effective properties for the multiscale materials, Dynamic Shear Rheometer (DSR) is used to measure the dynamic shear moduli of asphalt binder, mastic, and mortar, and Simple Performance Tester (SPT) is used to measure the axial dynamic modulus of asphalt mixture. Temperature and frequency sweep tests were applied to measure the dynamic modulus of the multiscale asphalt materials and the temperatures, frequencies, and loading mode for different tests are summarized in Table 3 [25].

It should be noted that the primary test results of the mixture are the axial dynamic modulus $\left|E^{*}\right|$ and Poisson's ratio, which were further used to predict the mixture dynamic shear modulus $\left|G^{*}\right|$. More details about this process can be found in the literature [25]. The master curves were obtained at the reference temperature of $15^{\circ} \mathrm{C}$ according to

$$
\log \left(\left|G^{*}\right|\right)=\alpha+\frac{\beta-\alpha}{1+10^{\left(f_{0}-\log (f)\right) p}},
$$

where $\left|G^{*}\right|=$ dynamic shear modulus $(\mathrm{Pa}) ; f=$ reduced frequency $(\mathrm{rad} / \mathrm{s}) ; \alpha, \beta, f_{0}$, and $p=$ fitting parameters.

The test results and master curves for multiscale asphalt materials were shown in Figure 3, and the fitting parameters of master curves were listed in Table 4.

3.2. Multiscale Validation of the Model's Applicability. According to finalized material parameters, the five micromechanical models were applied to predict the effective shear modulus of the multiscales asphalt materials, respectively. For the mastic scale, the binder is the matrix phase and the fillers are the particle phase. But for the mortar scale, the mastic is the matrix phase and there are two particle phases, which

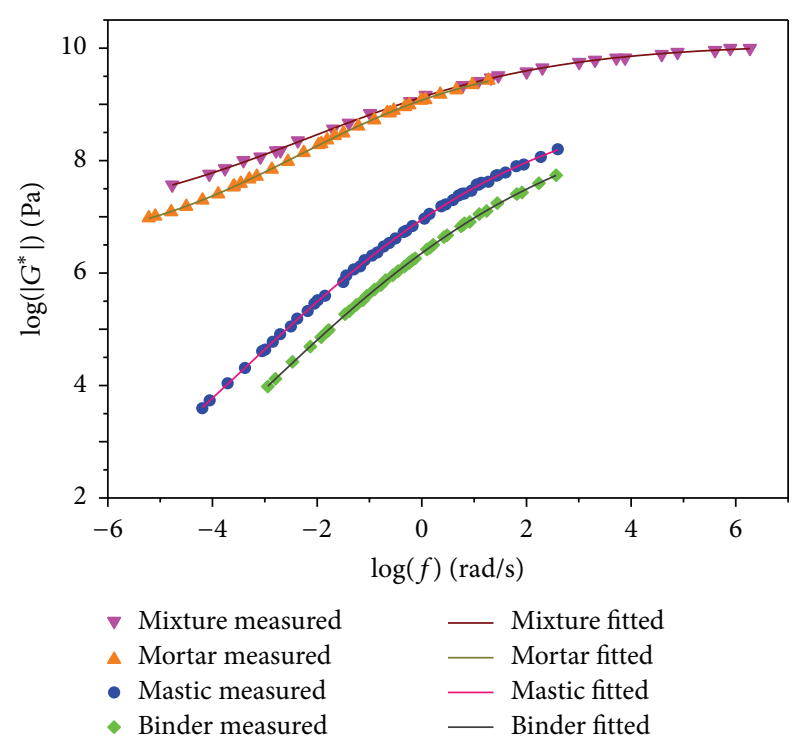

Figure 3: Experiment data and converted master curve of four materials.

are fine aggregates and voids, so a two-step approach was applied. The fine aggregates were firstly added to the mastic matrix for prediction, and then voids were added to the fine aggregate-mastic composite. The voids were considered as special kinds of particles whose modulus was assumed as zero. The effective modulus prediction for asphalt mixture is similar to the mastic while the mortar is considered as the matrix phase and the coarse aggregate as the particle phase. All of the effective moduli were predicted at 10 frequencies $\left(1.0 \times 10^{-5}\right.$ to $\left.1.0 \times 10^{5} \mathrm{rad} / \mathrm{s}\right)$ and compared with the measured values, as shown in Figures 4-6.

From Figures $4-6$, it is found that the micromechanical models are with different applicability for the three-scale asphalt materials. For the mastic scale, all of the five models can well predict the effective modulus across the whole frequency range, but the prediction accuracies for mortar and mixture scales were poorer and the prediction accuracies of the five models exhibit difference. For the mixture scale, all five models tend to overestimate at the intermediate frequencies but show good agreement at low and high frequencies except for the SC model. For the mortar scale, the TPS model was relatively better than others, and the effective modulus was a little overestimated at the higher frequencies (or lower temperatures) while it was underestimated at the lower frequencies (or higher temperatures). 


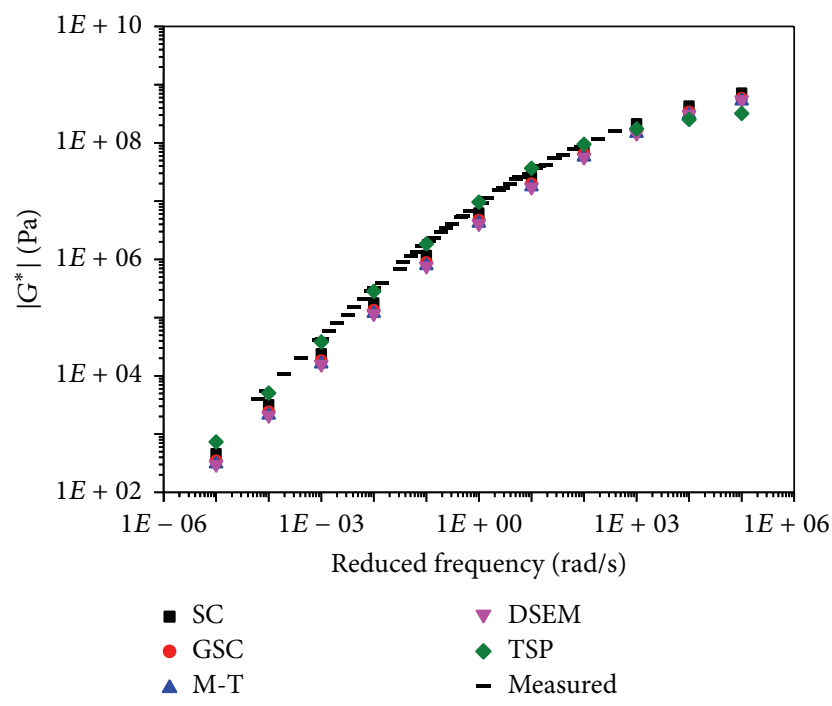

Figure 4: Predicted and measured dynamic shear modulus of mastic.

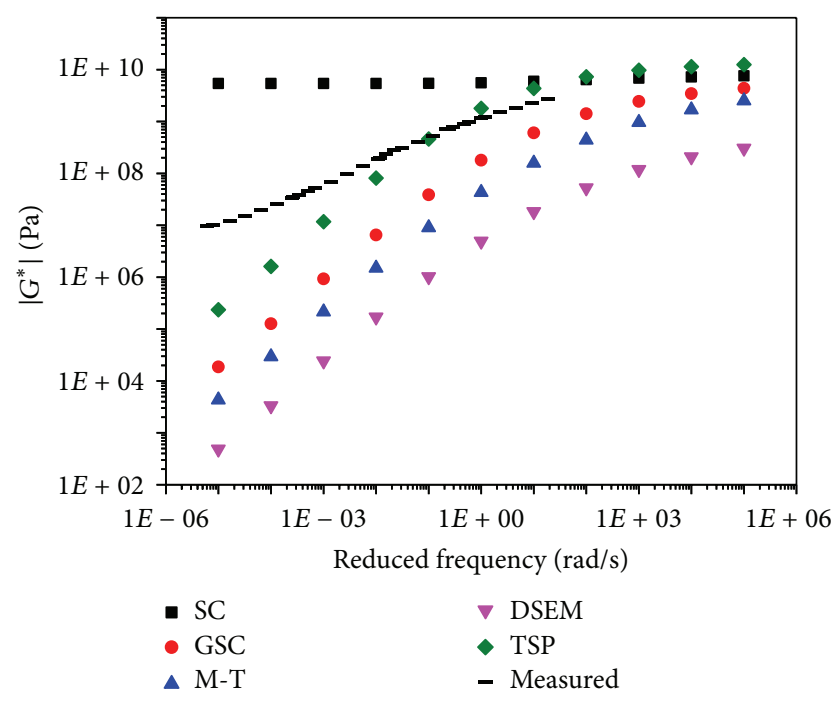

FIGURE 5: Predicted and measured dynamic shear modulus of mortar.

The good agreements of different models observed at the mastic scale owe to the low particle volume fraction of fillers in mastic. The reason for this is that, at low particle concentrations, the microstructure of composite can better match the assumptions of different models [3]. Compared with the mortar scale, the prediction accuracy of mixture scale is better. This is because the effective modulus prediction of mixture scale is based on the measured value of asphalt mortar and the lower particle volume fraction of coarse aggregates while there are two particle phases (fine aggregates and voids) with higher volume fraction in asphalt mortar.

Due to those reasons, the deviation between the predicted and measured values increases sharply for the mortar scale, especially at lower frequency and higher temperature. Moreover, at lower frequency and higher temperature, the modulus of asphalt matrix becomes smaller and the modulus

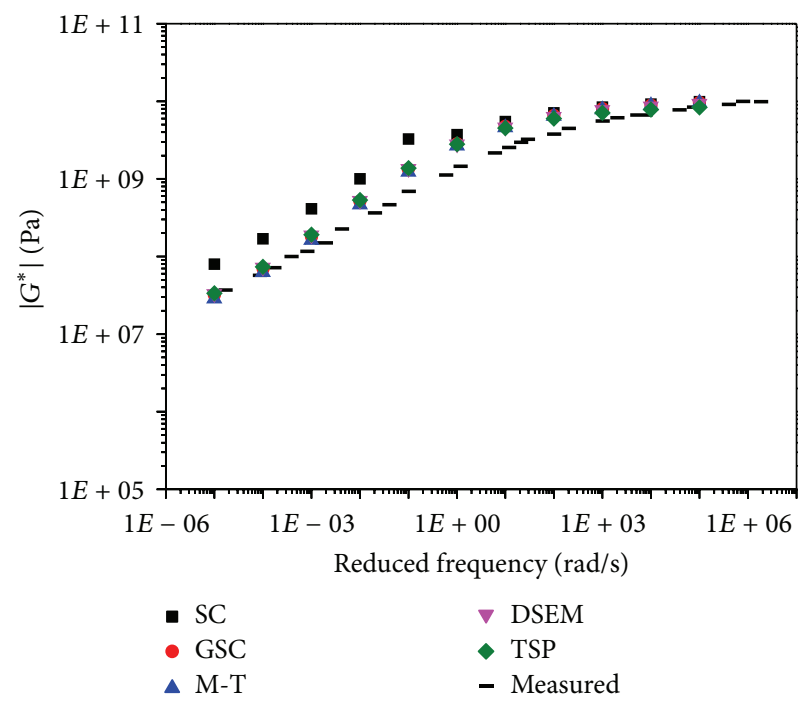

FIgURE 6: Predicted and measured dynamic shear modulus of mixture.

mismatch between the asphalt matrix and aggregate particles becomes bigger, which is the other important factor causing the deviation.

\section{Effects of Particle Volume Fraction and Modulus Mismatch}

4.1. Experiments of the Mastic-Scale Asphalt Materials. As discussed above, the complicated compositions of multiscale asphalt mixture challenge the applicability of micromechanical models, which have been also demonstrated by Buttlar and Roque [10], Li and Metcalf [11], Li et al. [20], and others. It is found that the higher particle volume fraction and the bigger modulus mismatch between particle and matrix were the two principle factors that deteriorate the applicability of the micromechanical models.

For further validation and evaluation of the effects of these two factors on the applicability of the micromechanical models, the dynamic shear moduli of mastic with different filler volume fraction were collected from the report by Underwood and Kim [27], and the master curves at the reference temperature of $15^{\circ} \mathrm{C}$ were obtained according to (1). The test results and converted master curves of asphalt mastic were shown in Figure 7, and the fitting parameters of master curves were listed in Table 3 . In Figure 7 and Table 5, the MS denotes mastic, and the numbers following MS denote the filler volume fractions in percentage.

4.2. Stiffening Effects of Aggregate Particles. Asphalt matrix is a kind of viscoelastic material, whose stiffness is much smaller than aggregate particle. When the aggregate particles are added to the asphalt matrix, the asphalt-aggregate composite system becomes stiffer due to the stiffening effect of the aggregate particle. The higher the aggregate particles volume fraction is, the stronger the effect will be. This stiffening effect could be represented by the modulus ratio of the composite to the asphalt matrix. Figure 8 illustrates the relations between 


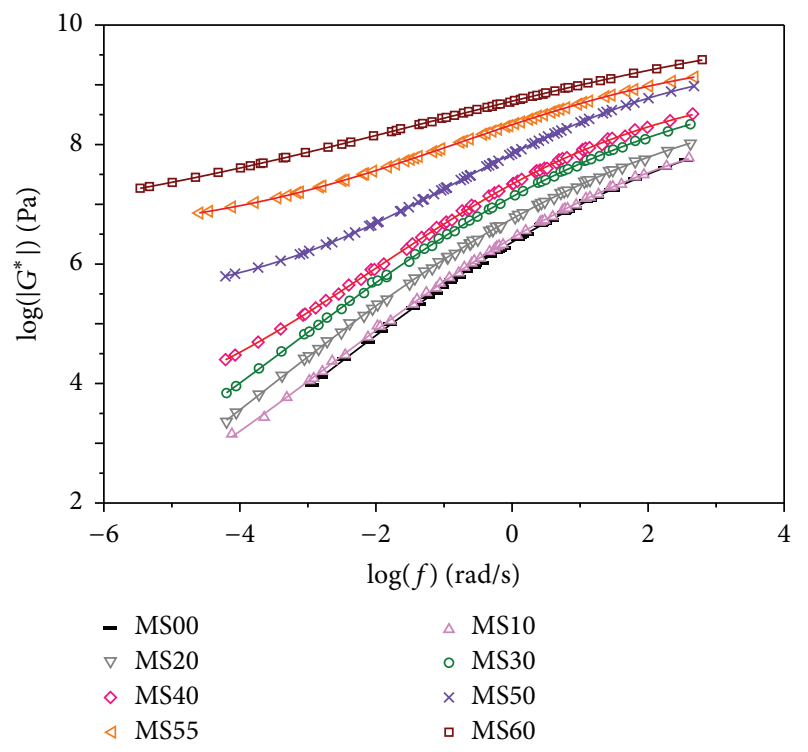

FIgURE 7: Experiment data and converted master curve of mastic.

TABLE 5: Fitting parameters of the master curve of mastic.

\begin{tabular}{lccccc}
\hline $\begin{array}{l}\text { Asphalt } \\
\text { mastic }\end{array}$ & $\alpha$ & $\beta$ & $f_{0}$ & $p$ & $R^{2}$ \\
\hline MS00 & -0.948 & 9.049 & -2.871 & 0.152 & 0.99 \\
MS10 & -0.136 & 8.827 & -2.637 & 0.1662 & 0.99 \\
MS20 & -1.693 & 9.241 & -3.759 & 0.14 & 0.99 \\
MS30 & -0.106 & 9.348 & -3.276 & 0.156 & 0.99 \\
MS40 & 2.638 & 9.167 & -2.008 & 0.202 & 0.99 \\
MS50 & 5.245 & 9.519 & -0.791 & 0.242 & 0.99 \\
MS55 & 6.426 & 9.624 & -0.791 & 0.213 & 0.99 \\
MS60 & 6.291 & 10.374 & -1.374 & 0.123 & 0.99 \\
\hline
\end{tabular}

the modulus ratio and the filler volume fraction of asphalt mastic under three test frequencies $(0.01,10$, and $50 \mathrm{~Hz})$. It is found that the modulus ratio firstly linearly and then exponentially increases with the increasing filler volume fraction, and the modulus ratio at lower frequency is stronger for asphalt mastic with the same filler volume fraction.

According to the researches by Faheem and Bahia [28, 29], the stiffening effect of filler particles could be divided into two zones, which are the diluted and concentrated zones. Within the diluted zone, the filler volume fraction is small. The filler particles are uniformly suspended in the asphalt matrix and do not contact each other. In this case, the microstructure of asphalt mastic is well in accordance with the hypotheses of micromechanical models and the predictions of effective modulus should be good. However, the filler volume fraction increases to a high level within the concentrated zone, and the predictions accuracy will be deteriorated. The deviation maybe resulted from the ignored particle interaction. At a low filler volume fraction, the interactions between fillers are very limited and contribute little to the stiffening effect. As the filler volume fraction

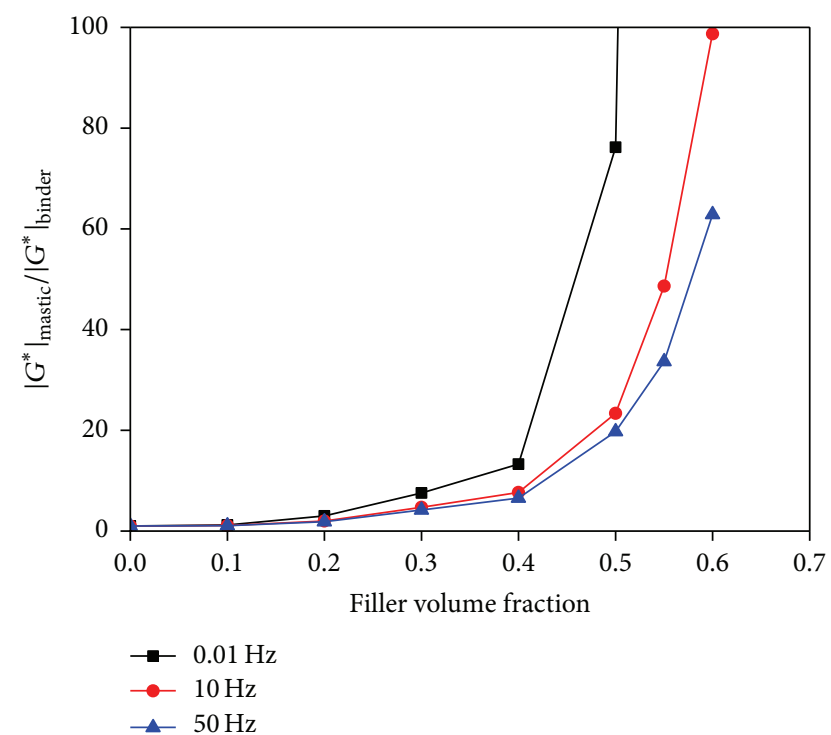

FIgURE 8: Relation between modulus ratio and filler volume fraction.

increases, the particle interaction contributes more to the stiffening effect.

It should be noted that the viscoelastic property of asphalt matrix also affects the prediction accuracy due to the modulus mismatch between the matrix and reinforcement particles. For the asphalt mastic with a fixed filler volume fraction, the smaller the modulus mismatch is at higher frequency or lower temperature, the better the prediction accuracy will be.

4.3. Effects of Particle Volume Fraction on the Prediction Accuracy. The effects of filler volume fraction on the accuracy of different models were shown in Figure 9 and all comparisons were conducted at a fixed frequency, $10 \mathrm{rad} / \mathrm{s}$. It is found that all models give a good prediction at low volume fraction of about $30 \%$, and the SC model tends to overestimate while others tend to underestimate with the increasing volume fraction. The higher the fraction is, the greater the deviation will be. Relatively, the SC model and GSC model are more suitable at high particle fraction.

To give a quantitative comparison, the double logarithmic linear regression method was adopted for each model in Figure 9 with the double logarithmic equation expressed as

$$
\log \left(\left|G_{\text {predicted }}^{*}\right|\right)=a+b \log \left(\left|G_{\text {measured }}^{*}\right|\right)
$$

where $\left|G_{\text {predicted }}^{*}\right|=$ predicted modulus, $\left|G_{\text {measured }}^{*}\right|=$ measured modulus, $a=$ intercept, and $b=$ slope.

The fitting parameters are listed in Table 6. The slope $b$ could be used as an index to evaluate the applicability of the models at high volume fraction, and the value of more approaching 1 means the better applicability. The same conclusion can be deduced as above based on the fitting results of the parameter $b$. Obviously, the SC and GSC models are again proved to be more applicable.

4.4. Effects of Modulus Mismatch on the Prediction Accuracy. Furthermore, the GSC model was selected to analyze the 


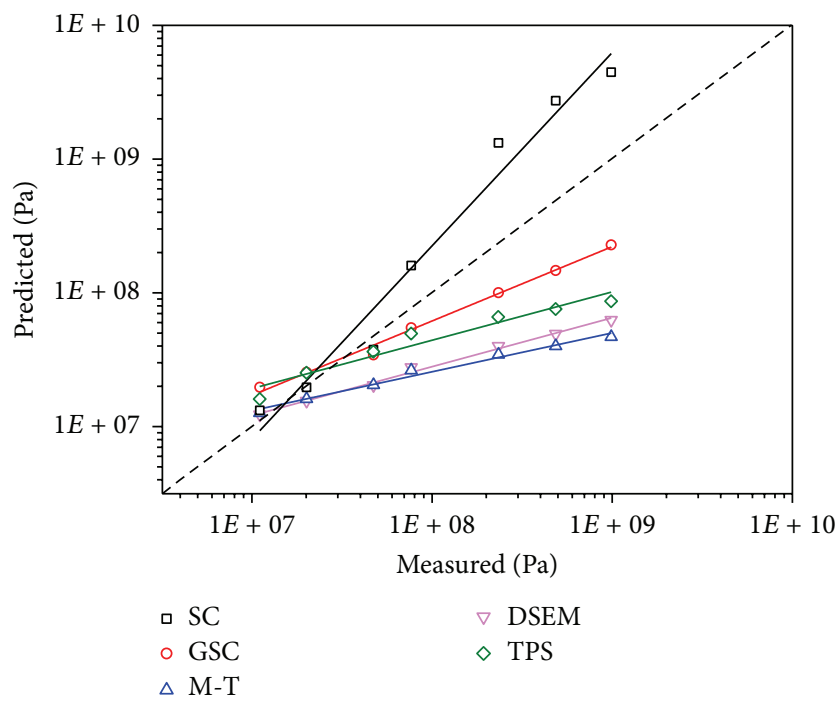

FIGURE 9: Effect of filler volume fraction on the prediction accuracy.

TABLE 6: Fitting parameters at $10 \mathrm{~Hz}$.

\begin{tabular}{lccccc}
\hline Parameters & SC & M-T & GSC & DSEM & TPS \\
\hline$b$ & 1.443 & 0.29 & 0.555 & 0.365 & 0.36 \\
$a$ & -3.189 & 5.091 & 3.349 & 4.531 & 4.76 \\
$R^{2}$ & 0.96 & 0.98 & 0.99 & 0.99 & 0.93 \\
\hline
\end{tabular}

TABLE 7: Fitting parameters of the GSC model at different frequencies.

\begin{tabular}{lccccc}
\hline Parameters & 0.01 & 0.1 & 1 & 10 & 50 \\
\hline$b$ & 0.327 & 0.398 & 0.476 & 0.555 & 0.605 \\
$a$ & 3.531 & 3.644 & 3.587 & 3.349 & 3.148 \\
$R^{2}$ & 0.98 & 0.98 & 0.99 & 0.99 & 0.99 \\
\hline
\end{tabular}

effect of modulus mismatch on the accuracies at different frequencies, as shown in Figure 10. The relationship between measured values and GSC predicted values was analyzed in the double logarithmic linear regression, and regressions results are listed in Table 7. Also, the parameter $b$ was used to evaluate the applicability of the GSC model at different frequencies. It is found that frequency (or temperature) significantly affects the accuracy of GSC model, especially within high particle volume fraction. The higher the frequency (or the lower the temperature) is, the better the model applicability will be. Though the other models are not analyzed, we think that the conclusions arrived at for GSC model are also suitable for other models.

\section{Conclusions}

There are so many micromechanical models for asphalt mixture, but the applicability is different due to the complicated compositions of asphalt mixture. In this paper, the applicability of some micromechanical models is validated from the collected and tested shear modulus of the multiscale

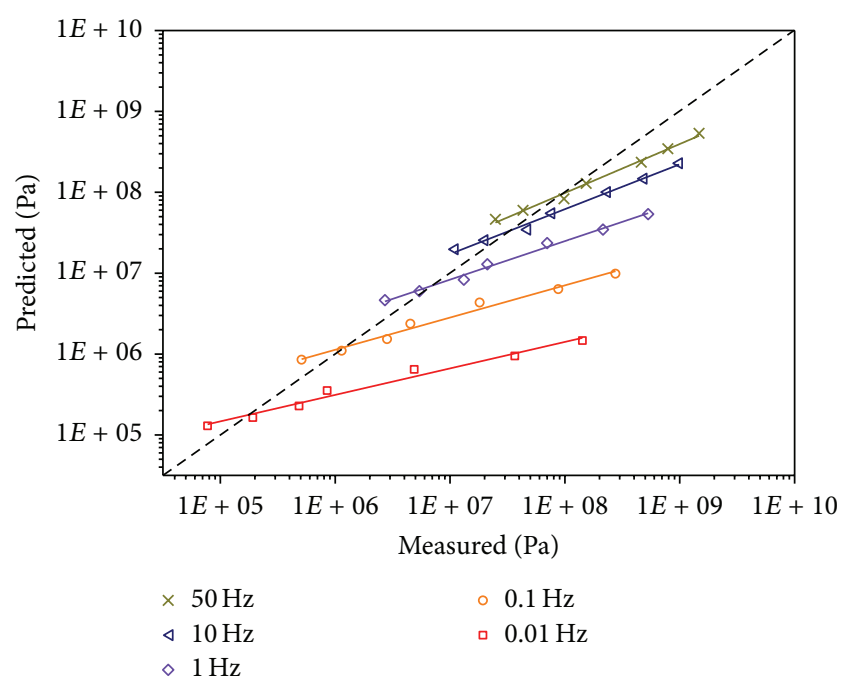

FIGURE 10: Effect of modulus mismatch on the prediction accuracy.

asphalt materials, including the SC, GSC, M-T, DSEM, and TPS models. Conclusions could be obtained as follows.

(1) All of the five models are applicable for the mastic scale, but the prediction accuracies for mortar and mixture scales are poorer. For the mixture scale, all models tend to overestimate at the intermediate frequencies but show good agreement at low and high frequencies except for the SC model. The TPS model is relatively better than others for the mortar scale.

(2) The applicability of micromechanical models is challenged due to the high particle volume fraction in the multiscale asphalt materials. With the filler volume fraction lower than $30 \%$, the filler particles could be uniformly suspended in the asphalt matrix and do not contact each other. However, the particle interaction contributes more to the stiffening effect within higher fraction, and the prediction is then deteriorated.

(3) Because asphalt matrix is viscoelastic materials and its modulus is smaller than aggregate particles, the modulus mismatch between matrix and particles also deteriorates the prediction accuracy, especially at the lower frequencies (or higher temperature). The higher the frequency (or the lower the temperature) is, the better the model applicability will be.

\section{Conflict of Interests}

The authors declare that there is no conflict of interests regarding the publication of this paper.

\section{Acknowledgments}

This work was supported by the National Natural Science Foundation of China (nos. 51378073, 51408043), the Natural Science Foundation of Shaanxi Province (2014JQ7278), and Special Fund for Basic Scientific Research of Central College of Changan University (310821153502, 310821152003). 


\section{References}

[1] E. Masad, "X-ray computed tomography of aggregates and asphalt mixes," Materials Evaluation, vol. 62, no. 7, pp. 775-783, 2004.

[2] L. Wang, J. Y. Park, and Y. R. Fu, "Representation of real particles for DEM simulation using X-ray tomography," Construction and Building Materials, vol. 21, no. 2, pp. 338-346, 2007.

[3] X. L. Du and L. Jin, "A review on meso-mechanical method for studying the static-mechanical properties of concrete," Advances in Mechanics, vol. 41, no. 4, pp. 411-426, 2011.

[4] Z. You, Y. Liu, and Q. Dai, "Three-dimensional microstructuralbased discrete element viscoelastic modeling of creep compliance tests for asphalt mixtures," Journal of Materials in Civil Engineering, vol. 23, no. 1, pp. 79-87, 2011.

[5] R. Hill, "A self-consistent mechanics of composite materials," Journal of the Mechanics and Physics of Solids, vol. 13, no. 4, pp. 213-222, 1965.

[6] B. Budiansky, "On the elastic moduli of some heterogeneous materials," Journal of the Mechanics and Physics of Solids, vol. 13, no. 4, pp. 223-227, 1965.

[7] T. Mori and K. Tanaka, "Average stress in matrix and average elastic energy of materials with misfitting inclusions," Acta Metallurgica, vol. 21, no. 5, pp. 571-574, 1973.

[8] R. Christensen and K. Lo, "Solutions for effective shear properties in three phase sphere and cylinder models," Journal of the Mechanics and Physics of Solids, vol. 27, no. 4, pp. 315-330, 1979.

[9] R. Mclaughlin, "A study of the differential scheme for composite materials," International Journal of Engineering Science, vol. 15, no. 4, pp. 237-244, 1977.

[10] W. G. Buttlar and R. Roque, "Evaluation of empirical and theoretical models to determine asphalt mixtures stiffnesses at low temperature," Journal of the Association of Asphalt Paving Technologists, vol. 65, pp. 99-141, 1996.

[11] Y. Li and J. B. Metcalf, "Two-step approach to prediction of asphalt concrete modulus from two-phase micromechanical models," Journal of Materials in Civil Engineering, vol. 17, no. 4, pp. 407-415, 2005.

[12] H. M. Yin, W. G. Buttlar, G. H. Paulino, and H. D. Benedetto, "Assessment of existing micro-mechanical models for asphalt mastic considering viscoelastic effects," Road Materials and Pavement Design, vol. 9, no. 1, pp. 31-57, 2008.

[13] H. L. Duan, X. Yi, Z. P. Huang, and J. Wang, "A unified scheme for prediction of effective moduli of multiphase composites with interface effects. Part I: theoretical framework," Mechanics of Materials, vol. 39, no. 1, pp. 81-93, 2007.

[14] J. D. Eshelby, "The determination of the elastic field of an ellipsoidal inclusion and related problems," Proceedings of the Royal Society of London A, vol. 241, pp. 376-396, 1957.

[15] V. Kushnevsky, O. Morachkovsky, and H. Altenbach, "Identification of effective properties of particle reinforced composite materials," Computational Mechanics, vol. 22, no. 4, pp. 317-325, 1998.

[16] N. Shashidhar and A. Shenoy, "On using micromechanical models to describe dynamic mechanical behavior of asphalt mastics," Mechanics of Materials, vol. 34, no. 10, pp. 657-669, 2002.

[17] A. Norris, "A differential scheme for the effective moduli of composites," Mechanics of Materials, vol. 4, no. 1, pp. 1-16, 1985.

[18] X. Shu and B. S. Huang, "Predicting dynamic modulus of asphalt mixtures with differential method," Road Materials and Pavement Design, vol. 10, no. 2, pp. 337-359, 2009.
[19] M. Kim and W. G. Buttlar, "Differential scheme effective medium theory for hot mix asphalt $-E^{*}-$ prediction," Journal of Materials in Civil Engineering, vol. 23, no. 1, pp. 69-78, 2011.

[20] G. Q. Li, Y. Q. Li, J. B. Metcalf, and S. S. Pang, "Elastic modulus prediction of asphalt concrete," Journal of Materials in Civil Engineering, vol. 11, no. 3, pp. 236-241, 1999.

[21] X. Shu and B. S. Huang, "Dynamic modulus prediction of HMA mixtures based on the viscoelastic micromechanical model," Journal of Materials in Civil Engineering, vol. 20, no. 8, pp. 530538, 2008.

[22] X. Shu and B. Huang, "Micromechanics-based dynamic modulus prediction of polymeric asphalt concrete mixtures," Composites Part B: Engineering, vol. 39, no. 4, pp. 704-713, 2008.

[23] Y. Kim and D. N. Little, "Linear viscoelastic analysis of asphalt mastics," Journal of Materials in Civil Engineering, vol. 16, no. 2, pp. 122-132, 2004.

[24] N. W. Tschoegl, The Phenomenological Theory of Linear Viscoelastic Behavior, Springer, Berlin, Germany, 1989.

[25] B. S. Underwood and Y. R. Kim, "Experimental investigation into the multiscale behaviour of asphalt concrete," International Journal of Pavement Engineering, vol. 12, no. 4, pp. 357-370, 2011.

[26] F. P. Zhou, F. D. Lydon, and B. I. G. Barr, "Effect of coarse aggregate on elastic modulus and compressive strength of high performance concrete," Cement and Concrete Research, vol. 25, no. 1, pp. 177-186, 1995.

[27] B. S. Underwood and Y. R. Kim, "A four phase micromechanical model for asphalt mastic modulus," Mechanics of Materials, vol. 75, no. 8, pp. 13-33, 2014.

[28] A. F. Faheem and H. U. Bahia, "Conceptual phenomenological model for interaction of asphalt binders with mineral fillers," Journal of the Association of Asphalt Paving Technologists, vol. 78, pp. 679-720, 2009.

[29] A. F. Faheem and H. U. Bahia, "Modelling of asphalt mastic in terms of filler-bitumen interaction," Road Materials and Pavement Design, vol. 11, no. 1, pp. 281-303, 2010. 

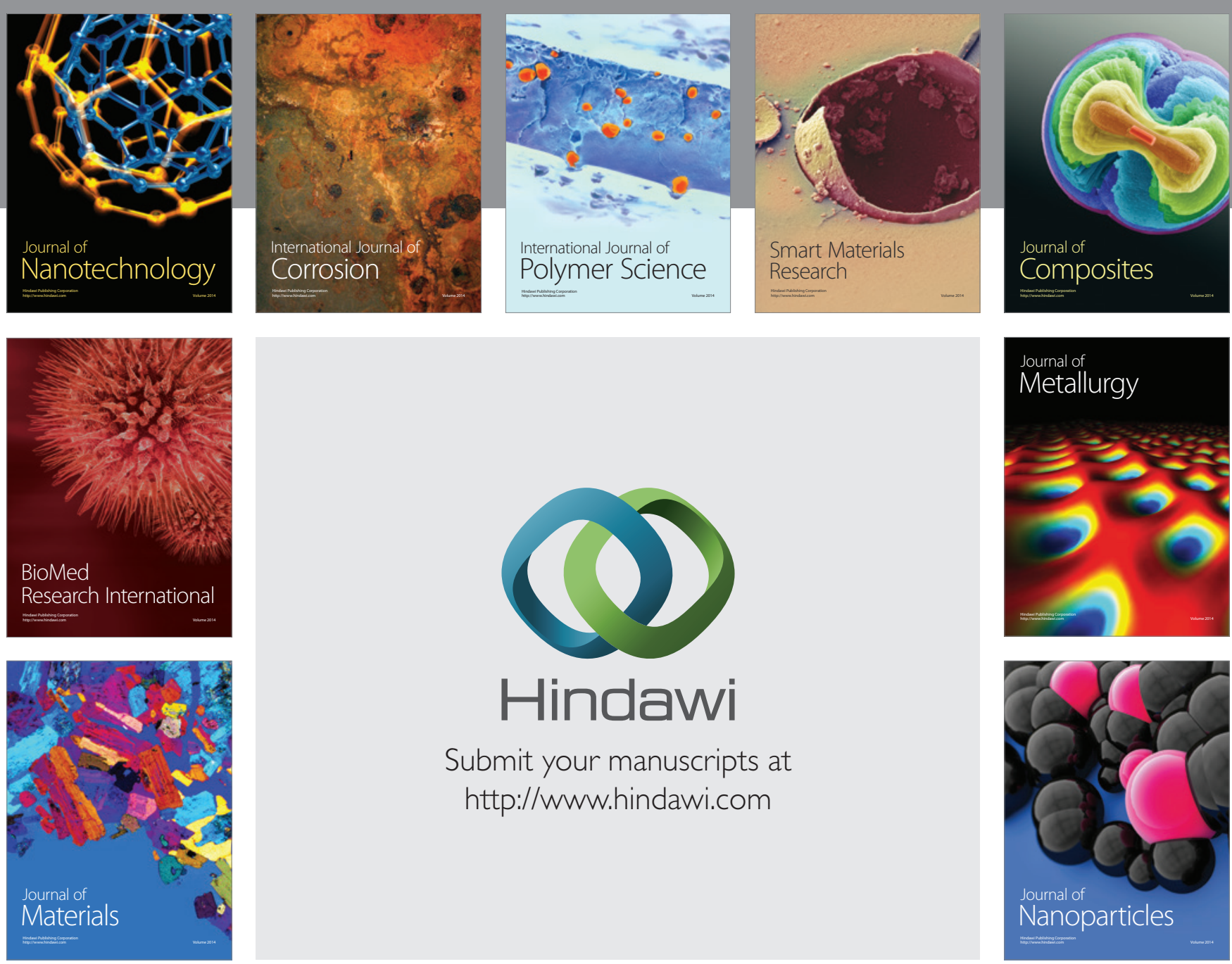

Submit your manuscripts at http://www.hindawi.com
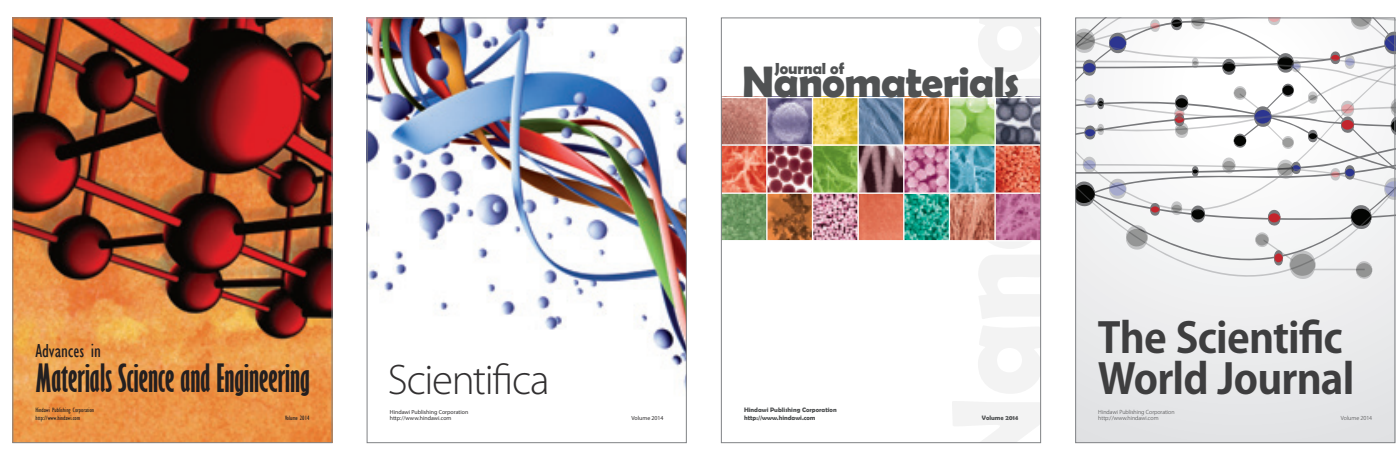

\section{The Scientific World Journal}
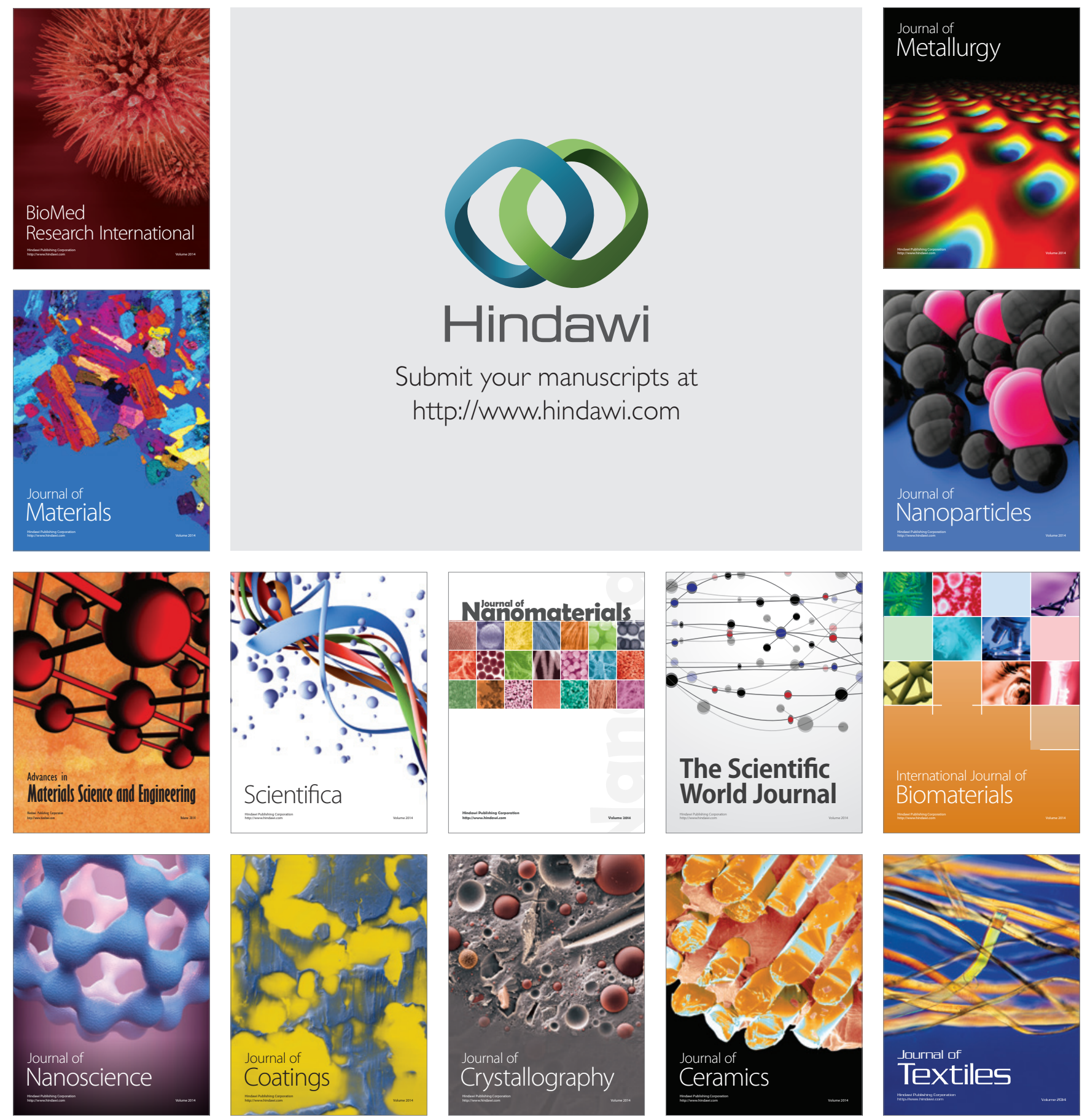